\title{
Uso de psicofármacos para síntomas neuropsiquiátricos en pacientes hospitalizados con COVID-19
}

Glauco Valdivieso-Jiménez*1,2,a

\section{RESUMEN}

La pandemia por la COVID-19 es la actual crisis sanitaria mundial que, hasta la fecha, ha cobrado miles de vidas en la mayoría de los países. Desde inicios del 2020 se estudia el comportamiento epidemiológico y clínico de este virus, así como también se han planteado esquemas terapéuticos que logren eliminar el virus, per se, y sus complicaciones a nivel sistémico. Sin embargo, los pacientes hospitalizados por esta infección también presentan síntomas neuropsiquiátricos, por lo que el manejo farmacológico requiere de consideraciones especiales al momento de su prescripción. Los síntomas neuropsiquiátricos secundarios más comunes en un cuadro de COVID-19 son ansiedad, insomnio, ánimo deprimido, delirio y agitación. La elección de psicofármacos debe basarse en el principio de no generar más daño, y valorar el riesgo-beneficio, el perfil farmacológico, las posibles interacciones y condiciones médicas previas del paciente.

Palabras clave: Psicotrópicos; Hospitalización; Coronavirus; COVID-19 (Fuente: DeCS BIREME).

\section{Use of psychotropic drugs for neuropsychiatric symptoms in patients hospitalized with COVID-19}

\section{ABSTRACT}

COVID-19 pandemic is the current global health crisis that, to date, has claimed thousands of lives in most countries. Since the beginning of 2020, the epidemiological and clinical behavior of the SARS-CoV-2, as well as proposals for therapeutic schemes that address the elimination of this virus per se and its complications at the systemic level, have been studied. However, neuropsychiatric symptoms also occur in patients hospitalized for this infection, so pharmacological management requires special considerations at the time of prescription. The most common neuropsychiatric symptoms secondary to COVID-19 infection are anxiety, insomnia, depressed mood, delirium and agitation. The choice of psychotropic drugs requires to be based on the principle of not generating more harm, and assessing the risk-benefit, pharmacological profile, possible interactions and previous medical conditions of the patient.

Keywords: Psychotropic drugs; Hospitalization; Coronavirus; Coronavirus infections (Source: MeSH NLM).

1 Hospital de Emergencias Villa El Salvador, Servicio de Salud Mental. Lima, Perú.

2 Instituto Peruano para el Estudio y Abordaje Integral de la Personalidad (IPEP). Lima, Perú.

a Médico, Especialista en Psiquiatría.

* Autor corresponsal. 


\section{INTRODUCCIÓN}

En la actualidad, vivimos una crisis mundial sanitaria, la pandemia por la COVID-19 que, hasta la fecha, ha cobrado miles de vidas en la mayoría de los países. El 30 de enero de 2020, el Comité de Emergencia de la Organización Mundial de la Salud (OMS) declaró este brote como una emergencia de salud global debido al reciente incremento de casos en China y otros países. Desde entonces se estudia el comportamiento epidemiológico y clínico de este virus (1), y también se han propuesto esquemas terapéuticos que consigan la eliminación del virus per se y sus complicaciones sistémicas. Se han identificado alteraciones en distintos sistemas, sobre todo en el respiratorio, y también a nivel hematológico, dermatológico, hepático y en el sistema nervioso central. Sin embargo, los hallazgos sobre los síntomas neuropsiquiátricos durante la hospitalización de pacientes complicados son escasos, aunque van en aumento.

Este trabajo es una revisión del manejo farmacológico de los síntomas neuropsiquiátricos de los pacientes con COVID-19, dirigido a todo médico, sea o no especialista en Psiquiatría o Neurología. Estas consideraciones se basan en los hallazgos identificados hasta la fecha en los estudios realizados en todo el mundo; además, debido a la complejidad de los fármacos antibióticos, antivirales, corticoides y demás, las opciones de tratamiento para los síntomas neuropsiquiátricos se ven limitadas por las interacciones farmacológicas y condiciones médicas previas.

\section{ESTRATEGIA DE BÚSQUEDA}

Se realizó una revisión narrativa a partir de una búsqueda en Pubmed de todos los artículos publicados desde el 1 de enero de 2020 hasta el 28 de junio de 2020 que incluyeran los siguientes términos: psychopharmachology AND COVID-19 y neuropsychiatric AND COVID-19. Seleccionamos los artículos que contenían información relacionada con el uso de psicofármacos para síntomas neuropsiquiátricos en pacientes hospitalizados por COVID-19. Se realizó un cribado de los artículos elegibles y se revisaron los títulos y resúmenes de todos los artículos encontrados. De las cuarenta y una publicaciones encontradas, catorce se descartaron porque no tenían relación directa con el tema y se incorporaron dos libros de texto y dos herramientas de interacciones farmacológicas online.

\section{PANORAMA GENERAL DE LA COVID-19}

Actualmente, hay un brote mundial de un nuevo tipo de coronavirus SARS-CoV-2 (que causa la COVID-19), que se originó en Wuhan, China, y se ha extendido a otros 140 países, incluidos Japón, Corea e Italia. La Organización Mundial de la Salud (OMS) declaró que la COVID-19 se ha convertido en un problema de salud global, causando severos daños en el sistema respiratorio. La evidencia actual indica que este virus se propagó a los humanos a través de la transmisión de animales salvajes vendidos ilegalmente en el mercado mayorista de mariscos de Wuhan ${ }^{(1)}$.

Los síntomas típicos de COVID-19 son fiebre, dolor de garganta, fatiga, tos o disnea, junto con el antecedente de una exposición reciente. A partir del 16 de marzo de 2020, el brote de COVID-19 generó 168826 casos confirmados, incluidas 6503 muertes en todo el mundo, y esta cifra sigue aumentando (2). En el Perú, son más de 275000 los casos confirmados y más de 9000 fallecidos, hasta el 28 de junio del $2020^{(3)}$.

Se han realizado estudios para establecer el esquema terapéutico para los pacientes con esta infección, el cual incluye antivirales como el lopinavir/ritonavir, antibióticos (azitromicina), antiparasitarios (hidroxicloroquina), fármacos biológicos (tocilizumab), antiinflamatorios (corticoesteroides), antiparasitarios (ivermectina) ${ }^{(4,5)}$ y otros medicamentos de uso general. Ante la evidencia de síntomas neuropsiquiátricos en pacientes con COVID-19, es necesario tomar en consideración que la complejidad de estos planes terapéuticos originaría complicaciones en la práctica médica por las interacciones farmacológicas que se pueden generar con la prescripción de psicofármacos. ${ }^{(6-8)}$.

\section{COVID-19 Y SALUD MENTAL}

En una reciente revisión sistemática y metaanálisis, Rogers JPet al. ${ }^{(9)}$ incluyeron 3559 casos de pacientes con infección por MERS (del inglés, Middle East Respiratory Syndrome Coronavirus) y SARS COVID-19 (del inglés, Severe Acute Respiratory Syndrome Coronavirus) con la finalidad de identificar síntomas neuropsiquiátricos comunes. El estudio reveló que durante la fase aguda de la enfermedad, los pacientes hospitalizados presentaron síntomas de confusión ( $27,9 \%)$, ánimo deprimido (32,6\%), ansiedad (35,7\%), alteración de la memoria (34,1\%) e insomnio (41,9\%). Luego, en la etapa de postratamiento, se mantuvo el ánimo deprimido $(10,5 \%)$, el insomnio $(12,1 \%)$, la ansiedad $(12,3 \%)$, la irritabilidad $(12,8 \%)$, la alteración de la memoria $(18,9 \%)$, la fatiga $(19,3 \%)$ y las memorias traumáticas $(30,4 \%)$. En el grupo que ingresó a $\mathrm{UCl}$, se evidenció delirio (65\%) y agitación (69\%).

La investigación de Guo Q et al. (10) comparó el estado mental y los marcadores inflamatorios en 103 pacientes que estuvieron hospitalizados con síntomas leves de COVID-19. Los resultados de su estudio mostraron niveles elevados de depresión $(p<0,001)$, ansiedad $(p<0,001)$ y síntomas de estrés postraumático $(p<0,001)$. Por otro lado, los valores elevados de proteína C-reactiva (PCR), un marcador inflamatorio periférico, se correlacionaron positivamente con mayores índices de depresión $(p=0,003)$. 
En un hospital de cuidados paliativos, Lovell et al. (11) mostraron el impacto de los síntomas, el manejo y la respuesta al tratamiento en 101 pacientes con COVID-19: veinticuatro tuvieron delirio, y se identificó un grupo numeroso que presentaba diversas comorbilidades como hipertensión arterial, diabetes, demencia, cáncer de mama metastásico, enfermedad pulmonar crónica y falla renal, entre otras. Muchos de estos pacientes recibieron dosis únicas y combinadas de morfina, haloperidol y midazolam en infusión.

Liguori C et al. (12) estudiaron a 103 pacientes con infección por SARS-CoV-2. Noventa y cuatro pacientes $(91,3 \%)$ tuvieron, al menos, un síntoma neuropsiquiátrico. La alteración del sueño fue el síntoma más frecuente, seguido de disgeusia, dolor de cabeza, hiposmia y depresión. Las mujeres se quejaron con mayor frecuencia de hiposmia, disgeusia, mareos, parestesias, somnolencia diurna y dolor muscular. Estos pacientes también mostraron leucocitosis y niveles más bajos de proteína C-reactiva (PCR). Estos hallazgos de laboratorio se correlacionaron con la aparición de hiposmia, disgeusia, dolor de cabeza, somnolencia diurna y depresión.

\section{EFECTOS NEUROPSIQUIÁTRICOS DE ALGUNOS MEDICAMENTOS PARA COVID-19}

\section{Tocilizumab}

Los datos de pacientes con artritis reumática sugieren que tocilizumab puede tener algunos efectos positivos sobre los síntomas depresivos en la artritis reumatoide ${ }^{(13,14)}$. Sin embargo, Bilbul (34) menciona que una pequeña investigación, cuyos datos no fueron publicados, sugiere que los pacientes que recibieron tocilizumab después de un trasplante alogénico de células hematopoyéticas experimentaron un empeoramiento de los síntomas (depresión, ansiedad, dolor y sueño).

\section{Lopinavir/ritonavir}

La información farmacológica refiere que sus posibles efectos secundarios psiquiátricos pueden sueños anormales, agitación, ansiedad, confusión y labilidad emocional; aunque la información de casos o ensayos publicados con respecto a la incidencia de tales efectos es limitada ${ }^{(15)}$.

\section{Azitromicina}

Los efectos secundarios que reportados incluyen depresión psicótica, catatonia, delirio, reacción agresiva, ansiedad, mareos, dolor de cabeza, vértigo y somnolencia ${ }^{(16)}$.

\section{Corticoesteroides}

Los efectos secundarios neuropsiquiátricos de los corticosteroides han sido bien descritos en la literatura e incluyen depresión, manía, agitación, labilidad del estado de ánimo, ansiedad, insomnio, catatonia, despersonalización, delirio y psicosis. La mayoría de estos efectos aparecen en la etapa temprana del tratamiento, por lo general, en unos días, y la dosis es el factor de riesgo más significativo (por ejemplo, más de $40 \mathrm{mg} /$ día de prednisona) ${ }^{(17)}$.

\section{CONSIDERACIONES FARMACOLÓGICAS PARA EL MANEJO DE SÍNTOMAS NEUROPSIQUIÁTRICOS}

Debido a que la COVID-19 tiene un impacto en múltiples órganos, como el hígado, riñones, pulmones, y los sistemas cardiovascular, inmune y hematológico, el daño a estos niveles puede generar cambios farmacocinéticos en la absorción, la distribución, el metabolismo y la excreción de fármacos psicotrópicos; así también, pueden aparecer los efectos adversos propios de cada medicamento. Además, el esquema terapéutico para el manejo de la COVID-19 (antibióticos, antivirales, corticoides, anticoagulantes y sintomáticos) limita la posibilidad de indicar abiertamente la variedad de psicofármacos aprobados por la FDA para los síntomas psiquiátricos. Por ese motivo cada médico debe tener ciertas consideraciones para el empleo de un psicofármaco y los ajustes requeridos de las dosis cuando se emplean en pacientes hospitalizados por COVID-19.

\section{ANSIEDAD}

La ansiedad es un síntoma que se relaciona a una preocupación excesiva acerca del presente y el futuro, que altera las funciones biológicas como el apetito y el sueño, sobre todo nocturno. Además, se agregan manifestaciones somáticas evidentes, como temblores, sudoración, incremento de las frecuencias cardiaca y respiratoria, parestesias, entre otros. En el contexto de la cuarentena, es un síntoma que no solo aparece en la población infectada, sino en las personas con comorbilidades y en la población general.

El uso de benzodiacepinas para el manejo de la ansiedad puede producir un alivio inmediato; sin embargo, su uso debe restringirse con algunos fármacos de este grupo que bloqueen su efecto y provoquen efectos secundarios indeseables.

En nuestro medio, el uso de lorazepam, a diferencia de otras benzodiacepinas, puede ser beneficioso debido a que no presenta interacciones farmacológicas con hidroxicloroquina, lopinavir/ritonavir o tocilizumab. Por ello, el National Institute for Health and Care Excellence (NICE), en la guía Managing COVID-19 symptoms (including at the end of (ife) in the community, recomienda emplear 0,5 a $1 \mathrm{mg}$ de lorazepam, si es posible administrarlo por vía oral; si no es posible, se puede utilizar midazolam $(2,5$ a $5 \mathrm{mg}$ ) por vía subcutánea (18). En contraste, un consenso entre diversas sociedades médicas españolas, en las que se incluye la Sociedad de Psiquiatría Biológica, advierte acerca del uso de midazolam, en todas sus formas de administración, debido a las interacciones que tiene con 
lopinavir/ritonavir al incrementar sus concentraciones plasmáticas, aumentar el riesgo de sedación (inhibición metabolismo vía CYP3A4) y disminuir el efecto o niveles séricos del antiviral (19). Por otro lado, algunos autores consideran el uso de alprazolam, ya que a bajas dosis se puede emplear en pacientes con ansiedad y síntomas de pánico con dificultad respiratoria leve; además, los efectos son inmediatos, el metabolismo es rápido y se elimina con celeridad. Se prefiere el uso de benzodiacepinas de corta acción, a diferencia de aquellas cuyos metabolitos séricos circulan por mayor tiempo ${ }^{(20)}$. Hasta el momento, no se han reportado desenlaces fatales al utilizar estos fármacos, por lo que su empleo en cuadros respiratorios complicados debe reconsiderarse en los pacientes con COVID-19.

Las benzodiacepinas deben usarse con precaución en pacientes con enfermedades respiratorias agudas 0 crónicas subyacentes para evitar la depresión respiratoria y la insuficiencia respiratoria aguda precipitante. La ansiedad y la agitación pueden ser parte de la descompensación respiratoria aguda, y se agravarían aún más por la administración de ansiolíticos. Es importante evaluar cuidadosamente la etiología de la ansiedad y la agitación antes de considerar el uso de benzodiacepinas. En la evaluación, se puede incluir el estado mental del paciente (siempre que las condiciones médicas y de espacio lo permitan), el uso de los músculos accesorios de la respiración, los signos vitales y la saturación de oxígeno, así como solicitar pruebas de laboratorio y estudios de imágenes adicionales cuando esté clínicamente indicado. La vigilancia estrecha del estado respiratorio, cardiovascular y neurológico es primordial para detectar signos tempranos de deterioro clínico potencial por el uso de benzodiacepinas ${ }^{(20)}$.

Se ha comentado que el uso de gabapentina y pregabalina también podría ser seguro para el manejo de síntomas ansiosos leves y moderados, ya que no se evidencian interacciones con azitromicina, lopinavir/ritonavir, hidroxicloroquina, tocilizumab, ni ivermectina. A pesar de lo que reportan las investigaciones, no se han realizado estudios que prueben estos planteamientos ${ }^{(19-21)}$ (Tabla 1 ).

Tabla 1. Fármacos de elección para el manejo de ansiedad en pacientes hospitalizados por COVID-19 ${ }^{(19,21,22)}$

\begin{tabular}{|c|c|c|c|c|c|c|}
\hline \multirow{2}{*}{$\begin{array}{l}\text { Fármaco } \\
\text { de elección }\end{array}$} & \multicolumn{5}{|c|}{ Interacción farmacológica } & \multirow[t]{2}{*}{ Observaciones } \\
\hline & $\begin{array}{l}\text { Lopinavir/ } \\
\text { Ritonavir } \\
\end{array}$ & Hidroxicloroquina & Tocilizumab & Ivermectina & Azitromicina & \\
\hline Lorazepam & No & No & No & No & No & $\begin{array}{l}\text { Uso por vía } \\
\text { oral. } \\
\text { Precaución por } \\
\text { insuficiencia } \\
\text { respiratoria. }\end{array}$ \\
\hline Alprazolam & $\begin{array}{l}\text { Ritonavir } \\
\text { aumenta el } \\
\text { nivel de } \\
\text { alprazolam y su } \\
\text { efecto a través } \\
\text { del } \\
\text { metabolismo } \\
\text { de CYP3A4. }\end{array}$ & No & No & No & No & $\begin{array}{l}\text { Uso por vía } \\
\text { oral. } \\
\text { Monitorizar de } \\
\text { cerca. Usar } \\
\text { dosis bajas. }\end{array}$ \\
\hline Midazolam & $\begin{array}{l}\text { Ritonavir } \\
\text { aumenta } \\
\text { concentración } \\
\text { plasmática de } \\
\text { midazolam y el } \\
\text { riesgo sedación } \\
\text { (inhibición del } \\
\text { metabolismo } \\
\text { vía CYP3A4). }\end{array}$ & No & $\begin{array}{l}\text { Moderado. } \\
\text { Disminuye } \\
\text { concentraciones } \\
\text { plasmáticas de } \\
\text { midazolam. }\end{array}$ & $\begin{array}{l}\text { Aumenta los } \\
\text { niveles de } \\
\text { ivermectina } \\
\text { mediante } \\
\text { transportador } \\
\text { p-glicoproteina } \\
\text { MDR1. }\end{array}$ & $\begin{array}{l}\text { No } \\
\text { a } \\
\end{array}$ & $\begin{array}{l}\text { Uso por vía } \\
\text { subcutánea,re } \\
\text { comendado } \\
\text { por la guía } \\
\text { NICE (17). }\end{array}$ \\
\hline
\end{tabular}




\begin{tabular}{|c|c|c|c|c|c|c|}
\hline \multirow{2}{*}{$\begin{array}{c}\text { Fármaco } \\
\text { de elección }\end{array}$} & \multicolumn{5}{|c|}{ Interacción farmacológica } & \multirow[t]{2}{*}{ Observaciones } \\
\hline & $\begin{array}{l}\text { Lopinavir/ } \\
\text { Ritonavir }\end{array}$ & Hidroxicloroquina & Tocilizumab & Ivermectina & Azitromicina & \\
\hline & $\begin{array}{l}\text { Efecto grave } \\
\text { con inhibidores } \\
\text { de la proteasa. } \\
\text { Vías oral y } \\
\text { parenteral. } \\
\text { Disminuye } \\
\text { efecto o nivel } \\
\text { de ritonavir } \\
\text { mediante } \\
\text { transportador } \\
\text { p-glicoproteina } \\
\text { MDR1. }\end{array}$ & & & & & \\
\hline Gabapentina & No & No & No & No & No & $\begin{array}{l}\text { Uso por vía } \\
\text { oral. Útil para } \\
\text { ansiedad, } \\
\text { insomnio y } \\
\text { agitación. } \\
\text { Usar en dosis } \\
\text { bajas para } \\
\text { síntomas leves } \\
\text { o moderados. }\end{array}$ \\
\hline Pregabalina & No & No & No & No & No & $\begin{array}{l}\text { Uso por vía } \\
\text { oral. Útil para } \\
\text { ansiedad, } \\
\text { insomnio y } \\
\text { agitación. } \\
\text { Emplear en } \\
\text { dosis bajas } \\
\text { para síntomas } \\
\text { leves o } \\
\text { moderados. }\end{array}$ \\
\hline
\end{tabular}

NICE: National Institute for Health and Care Excellence (2020)

Fuente: Autoría propia.

\section{INSOMNIO}

El insomnio es definido como la dificultad para conciliar el sueño nocturno y que trae consigo complicaciones somáticas y cognitivas a corto, mediano y largo plazo (22).

Como se ha visto en estudios referentes a síntomas neuropsiquiátricos en la población infectada con COVID19, el insomnio es frecuente, por lo que su manejo debe ser ampliamente considerado. Si bien es cierto que este síntoma puede ser un trastorno previo, también puede acompañar a la ansiedad por estar en un ambiente hospitalario. Se puede asumir que, si el insomnio se origina en la ansiedad, 
se podría considerar el uso de benzodiacepinas y otros fármacos con efectos hipnóticos ${ }^{(23)}$.

Algunos antidepresivos con mecanismos atípicos, como la mirtazapina, pueden ser utilizados como hipnóticos, sobre todo en los pacientes con disminución de apetito adicional, como en la población geriátrica. El consenso español reporta que el uso de mirtazapina tiene moderado riesgo al combinarse con lopinavir/ritonavir, ya que se incrementan los niveles de mirtazapina por la vía CYP3A4, y el efecto sobre el QT aditivo. El ritonavir incrementa los niveles de mirtazapina (por la vía CYP3A4, 2D6 y/o 3A4), lo que favorece que aparezcan sus propios efectos secundarios en un $50 \%$. Según las plataformas de interacción farmacológica, se concluye que no existe contraindicación absoluta, pero puede utilizarse con dosificaciones bajas del $50 \%$, vigilancia de la sedación y del efecto respiratorio.
El riesgo de prolongación del intervalo QT puede ser dependiente de la dosis. No existen mayores hallazgos en estudios de población hospitalaria para el manejo de insomnio ${ }^{(19,23)}$.

En algunos estudios se ha mencionado que la melatonina podría ser una opción en el esquema general de la enfermedad. Tiene propiedades antinflamatorias y antioxidantes, reduce la permeabilidad de los vasos, disminuye la ansiedad, limita el uso de sedantes y mejora la calidad del sueño, por lo que es efectiva en pacientes de cuidados críticos y podría ser beneficiosa para mejorar los resultados clínicos en los pacientes con COVID-19 $(24,26)$. La melatonina tiene un alto perfil de seguridad y se ha reportado que limita las enfermedades relacionadas con el virus de manera significativa ${ }^{(23-26)}$ (Tabla 2).

Tabla 2. Fármacos de elección para el manejo de insomnio en pacientes hospitalizados por COVID-19 ${ }^{(19,21,22)}$

\begin{tabular}{|c|c|c|c|c|c|c|}
\hline \multirow{2}{*}{$\begin{array}{l}\text { Fármaco } \\
\text { de elección }\end{array}$} & \multicolumn{4}{|c|}{ Interacción farmacológica } & \multicolumn{2}{|r|}{ Observaciones } \\
\hline & $\begin{array}{l}\text { Lopinavir/ } \\
\text { Ritonavir }\end{array}$ & Hidroxicloroquina & Tocilizumab & Ivermectina & Azitromicina & \\
\hline Mirtazapina & $\begin{array}{l}\text { Lopinavir aumenta } \\
\text { el nivel de } \\
\text { mirtazapina vía } \\
\text { CYP3A4 y efecto } \\
\text { sobre el QT aditivo. } \\
\text { Ritonavir eleva } \\
\text { niveles de } \\
\text { mirtazapina vía } \\
\text { CYP3A4 1A2, 2D6 } \\
\text { y/o 3A4. } \\
\text { El efecto eleva en } \\
50 \text { \% los niveles de } \\
\text { mirtazapina. }\end{array}$ & $\begin{array}{l}\text { Aumento QT } \\
\text { leve, efecto } \\
\text { teórico y de } \\
\text { escasa } \\
\text { significación } \\
\text { clínica. }\end{array}$ & No & No & No & $\begin{array}{l}\text { Uso por vía oral. } \\
\text { Reducir dosis al } \\
50 \% \text {. } \\
\text { Inicio en } 7,5 \mathrm{mg} \text {, } \\
\text { máximo } 15 \mathrm{mg} \text {. } \\
\text { Vigilar sedación y } \\
\text { efecto respiratorio } \\
\text { El efecto QT es } \\
\text { dependiente de la } \\
\text { dosis. }\end{array}$ \\
\hline Melatonina & No & No & No & No & No & Uso por vía oral \\
\hline
\end{tabular}

Fuente: Autoría propia.

\section{DEPRESIÓN}

La depresión, al igual que la ansiedad, es una respuesta natural a un cambio repentino de circunstancias que implican separación e incertidumbre. La depresión se manifiesta por un estado de ánimo triste (deprimido), pérdida del placer $o$ interés en actividades que, de otra manera, serían placenteras; además, aparecen una variedad de síntomas como problemas con el apetito, el sueño, la energía, la concentración, sensación de inutilidad, culpa, desesperanza y también ideas suicidas ${ }^{(23)}$. El tratamiento farmacológico de la depresión debe considerarse según la severidad clínica, los efectos secundarios de los medicamentos, sus interacciones con otros fármacos y el tiempo necesario en el que se espera el efecto antidepresivo ${ }^{(23)}$.

En los casos en que los síntomas depresivos estén acompañados de ansiedad e insomnio, se justificaría el empleo de agentes con antagonismo del receptor de serotonina 5-HT2A, como la mirtazapina, incluso si el tratamiento se puede suspender antes del inicio de los efectos antidepresivos debido a un cambio en las circunstancias. El uso de mirtazapina puede ser particularmente apropiado para pacientes que, además 
del insomnio y los síntomas depresivos, muestren poco apetito, con o sin náuseas, debido a su antagonismo $\mathrm{H} 1 \mathrm{y}$ antagonismo 5-HT3. Se ha descrito que la mirtazapina podría interactuar con fármacos del esquema de tratamiento para COVID-19, por lo que es necesario considerar su mecanismo farmacocinético ${ }^{(27-32)}$.

Si los pacientes tienen un alto riesgo de delirio, el uso de antidepresivos debe evaluarse. Los antidepresivos tradicionales como los tricíclicos (ATC), por lo general, se evitan debido a sus propiedades anticolinérgicas, ya que empeoran los síntomas de delirio ${ }^{(23)}$.

No se han reportado estudios que aclaren el uso de antidepresivos específicos durante la hospitalización de un paciente con COVID-19, por lo que se tomarían en cuenta las consideraciones ya mencionadas. En una revisión en las herramientas de verificación de interacciones farmacológicas, observamos que el empleo de inhibidores selectivos de la recaptación de serotonina (ISRS) estaría parcialmente restringido por estas complicaciones. Podría usarse sertralina en dosis reducidas y estricta monitorización por su riesgo moderado de las condiciones que puedan alargar el QTc, sobre todo al combinarse con azitromicina ${ }^{(21,22)}$. Elempleo de fluoxetinay paroxetina junto con lopinavir/ritonavir se considera como una posibilidad, con monitorización cercana por el riesgo de incrementar los niveles séricos de sus metabolitos y potenciar sus efectos secundarios. Sin embargo, la combinación la sertralina y fluoxetina con hidroxicloroquina tiene un alto riesgo de incrementar el intervalo QTc ${ }^{(29,32,33)}$. Otros antidepresivos de reciente generación, como venlafaxina, desvenlafaxina y duloxetina, que están disponibles en nuestro país, pero no como parte del petitorio nacional, pueden utilizarse en dosis reducidas a un $50 \%{ }^{(27-29)}$, como se observa en la tabla 3.

Tabla 3. Fármacos de elección para el manejo de síntomas de depresión en pacientes hospitalizados por COVID-19 (19,21,22)

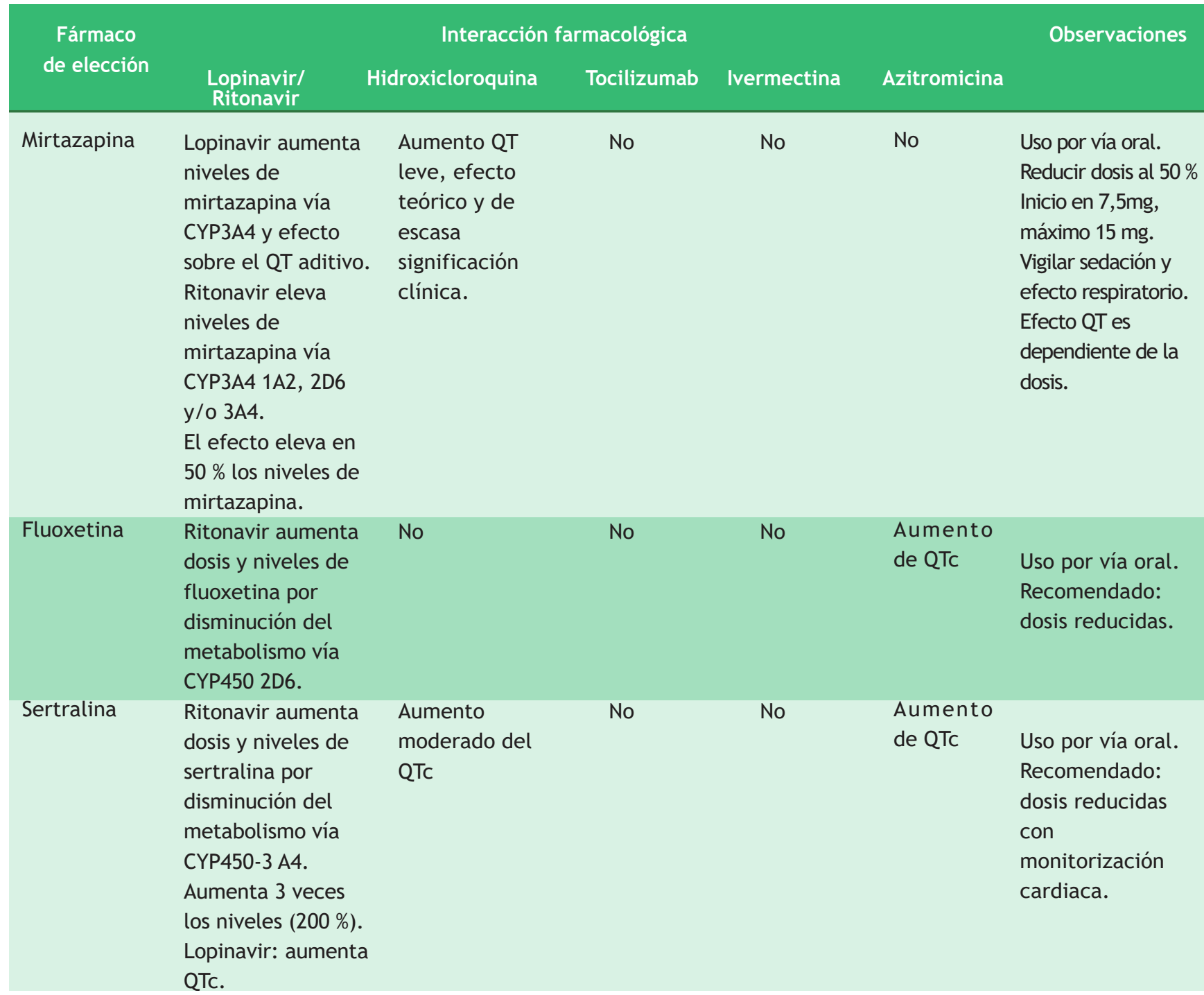




\begin{tabular}{|c|c|c|c|c|c|c|}
\hline \multirow{2}{*}{$\begin{array}{l}\text { Fármaco } \\
\text { de elección }\end{array}$} & \multicolumn{5}{|c|}{ Interacción farmacológica } & \multirow[t]{2}{*}{ Observaciones } \\
\hline & $\begin{array}{l}\text { Lopinavir/ } \\
\text { Ritonavir }\end{array}$ & Hidroxicloroquina & Tocilizumab & Ivermectina & Azitromicina & \\
\hline Paroxetina & $\begin{array}{l}\text { Ritonavir aumenta } \\
\text { dosis y niveles de } \\
\text { fluoxetina por } \\
\text { disminución del } \\
\text { metabolismo vía } \\
\text { CYP450 2D6. }\end{array}$ & No & No & No & $\begin{array}{l}\text { Aumento } \\
\text { de QTC }\end{array}$ & $\begin{array}{l}\text { Uso por vía oral. } \\
\text { Recomendado: } \\
\text { dosis reducidas. }\end{array}$ \\
\hline Venlafaxina & $\begin{array}{l}\text { Lopinavir/ritonavir } \\
\text { aumentan niveles } \\
\text { de venlafaxina } \\
\text { mediante } \\
\text { interacción } \\
\text { CYP-450-3A4. } \\
\text { Ritonavir: Mayor } \\
\text { interacción } \\
\text { metabolizadores } \\
\text { lentos CYP-450-2D6 } \\
\text { (7\% en caucásicos y } \\
2 \text { \% en } \\
\text { afroasiáticos). } \\
\text { Podría aumentar } \\
\text { niveles en 20 \% y } \\
70 \% \text { en } \\
\text { metabolizadores } \\
\text { lentos } 2 \text { D6. } \\
\text { Lopinavir: aumento } \\
\text { QTc. }\end{array}$ & $\begin{array}{l}\text { No } \\
\text { y } \\
6 \\
0\end{array}$ & No & No & $\begin{array}{l}\text { Aumento } \\
\text { de QTC }\end{array}$ & $\begin{array}{l}\text { Uso por vía oral. } \\
\text { Recomendado: } \\
\text { dosis reducidas. }\end{array}$ \\
\hline Desvenlafaxina & $\begin{array}{l}\text { Lopinavir/ritonavir } \\
\text { aumenta niveles de } \\
\text { venlafaxina } \\
\text { mediante } \\
\text { interacción } \\
\text { CYP-450-3A4. } \\
\text { Emplear con } \\
\text { precaución y } \\
\text { monitorizar. }\end{array}$ & e & No & No & No & $\begin{array}{l}\text { Uso por vía oral. } \\
\text { Recomendado: } \\
\text { dosis reducidas. }\end{array}$ \\
\hline Duloxetina & $\begin{array}{l}\text { Ritonavir puede } \\
\text { aumentar efecto de } \\
\text { duloxetina } \\
\text { mediante } \\
\text { interacción } \\
\text { CYP-450-2D6. }\end{array}$ & $\begin{array}{l}\text { Discreto } \\
\text { aumento de QTC } \\
\text { vía CYP 2D6 }\end{array}$ & No & No & No & $\begin{array}{l}\text { Uso por vía oral. } \\
\text { Recomendado: } \\
\text { dosis reducidas. }\end{array}$ \\
\hline
\end{tabular}

Fuente: elaboración propia. 


\section{AGITACIÓN/DELIRIO}

En pacientes con COVID-19, el delirio puede ser una manifestación de invasión directa del sistema nervioso central (SNC), la inducción de mediadores inflamatorios del SNC, un efecto secundario de otra falla del sistema orgánico, un efecto de estrategias sedantes, tiempo de ventilación mecánica prolongado o factores ambientales, que incluyen el aislamiento social (23). A partir de la experiencia con otros virus estrechamente relacionados de la familia Coronaviridae, la invasión directa del SNC parece ocurrir raramente y tarde en el curso de la enfermedad, pero puede estar asociada con convulsiones, alteraciones de la conciencia o signos de aumento de la presión intracraneal ${ }^{(30)}$.

La guía NICE recomienda que, si está disponible la vía oral para el paciente, se le administre haloperidol 0,5-1 mg por la noche, y cada 2 horas si es necesario; la dosis puede incrementarse hasta los $10 \mathrm{mg}$ diarios en adultos jóvenes o hasta $5 \mathrm{mg}$ en adultos mayores. Si no está disponible la vía oral, es necesaria la vía parenteral subcutánea o en infusión por 24 horas. Además, se puede considerar el uso adicional de benzodiacepinas como midazolam ${ }^{(18)}$.

Baller et al. recomiendan el uso de antipsicóticos atípicos, como risperidona, quetiapina, olanzapina y aripiprazol. Por otro lado, al revisar las interacciones farmacológicas, observamos que la quetiapina tiene un moderado efecto arritmogénico con alargamiento del QTC al combinarlo con lopinavir/ritonavir, hidroxicloroquina y azitromicina, pero puede utilizarse en dosis reducidas. Para los otros antipsicóticos no existen mayores contraindicaciones y se recomienda la administración de una dosis reducida solo si es imprescindible, además, vigilar si aparecen alteraciones en el electrocardiograma (ECG), mareos, palpitaciones, cefalea, disnea o síncope ${ }^{(31)}$. Estas consideraciones se amplían en la tabla 4.

Tabla 4. Fármacos de elección para el manejo de agitación/delirio en pacientes hospitalizados por COVID-19

\begin{tabular}{|c|c|c|c|c|c|c|}
\hline \multirow{2}{*}{$\begin{array}{c}\text { Fármaco } \\
\text { de elección }\end{array}$} & \multicolumn{5}{|c|}{ Interacción farmacológica } & \multirow[t]{2}{*}{ Observaciones } \\
\hline & $\begin{array}{l}\text { Lopinavir/ } \\
\text { Ritonavir }\end{array}$ & Hidroxicloroquina & Tocilizumab & Ivermectina & Azitromicina & \\
\hline Haloperidol & $\begin{array}{l}\text { Ritonavir + } \\
\text { haloperidol: ambos } \\
\text { aumentan el } \\
\text { intervalo QTc. } \\
\text { Ritonavir aumenta el } \\
\text { nivel o efecto de } \\
\text { haloperidol por } \\
\text { afectación del } \\
\text { metabolismo } \\
\text { hepático vía } \\
\text { CYP450-2D6 por } \\
\text { inhibición } \\
\text { competitiva por } \\
\text { ritonavir. }\end{array}$ & $\begin{array}{l}\text { Aumenta } \\
\text { intervalo QTc. }\end{array}$ & No & No & $\begin{array}{l}\text { Aumenta } \\
\text { intervalo } \\
\text { QTC. }\end{array}$ & $\begin{array}{l}\text { Alto riesgo de } \\
\text { aumento de QTC. } \\
\text { Uso por vía } \\
\text { subcutánea y oral, } \\
\text { recomendado por la } \\
\text { Guía NICE. Requiere } \\
\text { de monitorización } \\
\text { ECG continua. }\end{array}$ \\
\hline Risperidona & $\begin{array}{l}\text { Ritonavir aumenta } \\
\text { niveles de } \\
\text { risperidona y su } \\
\text { efecto mediante } \\
\text { interacción a nivel } \\
\text { transportador } \\
\text { p-glycoprotein } \\
\text { (MDR1). } \\
\text { Ritonavir aumenta } \\
\text { efecto de risperidona } \\
\text { mediante reducción }\end{array}$ & $\begin{array}{l}\text { Aumenta } \\
\text { intervalo QTC. }\end{array}$ & No & No & $\begin{array}{l}\text { Aumenta } \\
\text { intervalo } \\
\text { QTC. }\end{array}$ & $\begin{array}{l}\text { Uso por vía oral. } \\
\text { Dosis reducida, } \\
\text { máximo a } \\
0,5 \mathrm{mg} / \text { día. }\end{array}$ \\
\hline
\end{tabular}




\begin{tabular}{|c|c|c|c|c|c|c|}
\hline \multirow{2}{*}{$\begin{array}{c}\text { Fármaco } \\
\text { de elección }\end{array}$} & \multicolumn{5}{|c|}{ Interacción farmacológica } & \multirow[t]{2}{*}{ Observaciones } \\
\hline & $\begin{array}{l}\text { Lopinavir/ } \\
\text { Ritonavir }\end{array}$ & Hidroxicloroquina & Tocilizumab & Ivermectina & Azitromicina & \\
\hline & $\begin{array}{l}\text { de su metabolismo } \\
\text { por inhibición } \\
\text { competitiva } \\
\text { CYP450-2D6 por } \\
\text { ritonavir. } \\
\text { Lopinavir: Aumenta } \\
\text { QTc con efectos } \\
\text { aditivos. }\end{array}$ & & & & & \\
\hline Olanzapina & $\begin{array}{l}\text { Ritonavir disminuye } \\
\text { niveles de olanzapina } \\
\text { aumentando su } \\
\text { metabolismo (ajuste } \\
\text { dosis al alza) } \\
\text { mediante inducción } \\
\text { de enzimas } \\
\text { CYP450-1A2 y } \\
\text { uridin-5' difosfatoglu } \\
\text { curonosiltransferasa. }\end{array}$ & $\begin{array}{l}\text { Aumenta } \\
\text { intervalo QTC. }\end{array}$ & No & No & $\begin{array}{l}\text { Aumenta } \\
\text { intervalo } \\
\text { QTc. }\end{array}$ & $\begin{array}{l}\text { Recomendado inicio } \\
\text { con } 2,5 \mathrm{mg} \text { y subidas } \\
\text { de } 2,5 \text { en } 2,5 \mathrm{mg} \text {, } \\
\text { máx. } 15 \mathrm{mg} \text {. }\end{array}$ \\
\hline Quetiapina & $\begin{array}{l}\text { Ritonavir eleva el } \\
\text { nivel y efecto de } \\
\text { quetiapina mediante } \\
\text { interacción } \\
\text { metabolismo } \\
\text { CYP-450-3A4 y 2D6. } \\
\text { Lopinavir y } \\
\text { quetiapina aumentan } \\
\text { recíprocamente } \\
\text { toxicidad aditiva } \\
\text { sobre aumento QTc }\end{array}$ & $\begin{array}{l}\text { Aumenta } \\
\text { intervalo QTc. }\end{array}$ & No & No & $\begin{array}{l}\text { Aumenta } \\
\text { intervalo } \\
\text { QTC. }\end{array}$ & $\begin{array}{l}\text { Disminuir dosis a } 1 / 6 \\
\text { de dosis altas para } \\
\text { antecedentes de } \\
\text { trastornos psicóticos } \\
\text { crónicos. }\end{array}$ \\
\hline Aripiprazol & $\begin{array}{l}\text { Ritonavir eleva } \\
\text { niveles o efecto de } \\
\text { aripiprazol mediante } \\
\text { interacción del } \\
\text { metabolismo CYP3A4 } \\
\text { y CYP2D6. } \\
\text { Administración con } \\
\text { inhibidores de } \\
\text { CYP450-3A4 podría } \\
\text { aumentar las } \\
\text { concentraciones de } \\
\text { aripiprazol que se } \\
\text { metaboliza } \\
\text { parcialmente por esa } \\
\text { isoenzima. Podría }\end{array}$ & $\begin{array}{l}\text { Aumenta } \\
\text { intervalo QTC. }\end{array}$ & No & No & $\begin{array}{l}\text { Aumenta } \\
\text { intervalo } \\
\text { QTC. }\end{array}$ & $\begin{array}{l}\text { Dosis reducida al } \\
50 \% \text { con ritonavir, } \\
\text { pero no con el resto. } \\
\text { Dosis de inicio } 1 \text { mg } \\
\text { vía oral. }\end{array}$ \\
\hline
\end{tabular}




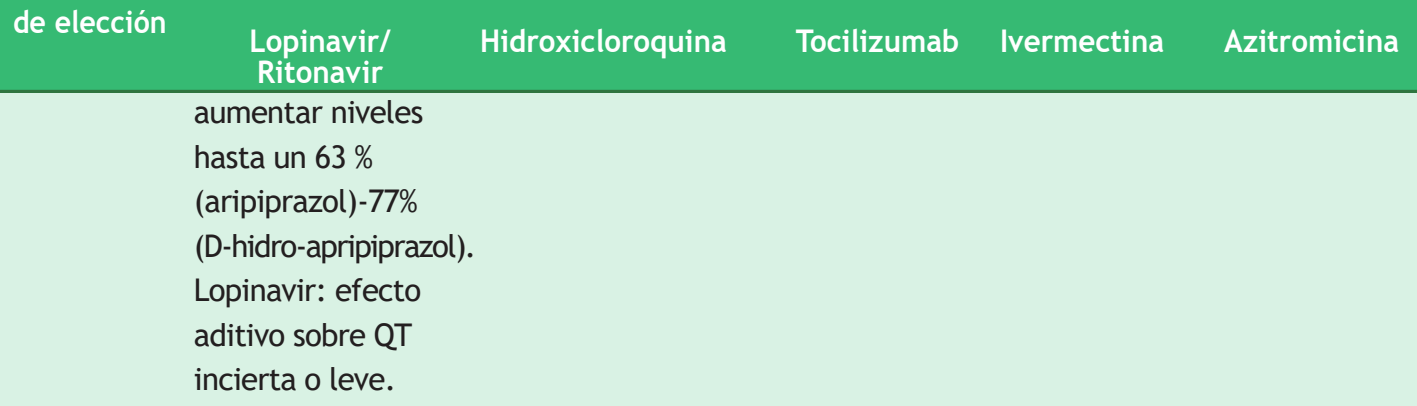

Fuente: elaboración propia.

\section{CONCLUSIONES}

Los estudios relacionados a la prevalencia e incidencia de trastornos mentales como una complicación de la COVID-19 son escasos, por lo que apenas se ha podido recolectar hallazgos en pocos participantes de muestras con síntomas neuropsiquiátricos, tanto en pacientes con complicaciones médicas, como por el uso de medicamentos complejos del esquema de tratamiento aprobado por guías internacionales y en el territorio nacional para COVID-19. De la revisión de los estudios disponibles, se concluye que los síntomas neuropsiquiátricos más comunes secundarios al padecimiento de la COVID-19 son ansiedad, insomnio, ánimo depresivo, delirio y agitación. Por lo tanto, la elección de psicofármacos para síntomas neuropsiquiátricos específicos debe basarse en el principio de no generar más daño y valorar el riesgo-beneficio, el perfil farmacológico, las posibles interacciones y las condiciones médicas previas del paciente. Esta información debería ser de conocimiento de todos los profesionales médicos que están manejando casos de pacientes con esta condición y no solamente de los especialistas en Psiquiatría o Neurología.

En el futuro, es necesario realizar nuevas investigaciones para ampliar la información sobre el uso de psicofármacos y síntomas neuropsiquiátricos que puedan complicar la evolución y pronóstico de pacientes hospitalizados por COVID-19.

Conflicto de intereses: El autor declara que no existe conflicto de intereses.

Fuentes de financiamiento: El artículo ha sido financiado por el autor.

\section{REFERENCIAS BIBLIOGRÁFICAS}

1. Velavan TP, Meyer CG. The COVID-19 epidemic. Trop Med Int Health. 2020; 25(3): 278-80.

2. Zhang L, Liu Y. Potential interventions for novel coronavirus in China: a systematic review. J Med Virol. 2020; 92(5): 479-90.

3. Gobierno del Estado Peruano. Coronavirus en el Perú: casos confirmados [Internet]. Lima; 2020. Disponible en: https://www.gob.pe/

4. Caly L, Druce JD, Catton MG, Jans DA, Wagstaff KM. The FDAapproved drug ivermectin inhibits the replication of SARS-CoV-2 in vitro. Antiviral Res. 2020; 178: 104787.

5. Choudhary R, Sharma AK. Potential use of hydroxychloroquine, ivermectin and azithromycin drugs in fighting COVID-19: trends, scope and relevance. New Microbes New Infect. 2020; 35: 100684.

6. Mehra MR, Desai SS, Ruschitzka F, Patel AN. Hydroxychloroquine or chloroquine with or without a macrolide for treatment of COVID-19: a multinational registry analysis. Lancet. 2020; S0140-6736(20): 31180-6.

7. Sanders JM, Monogue ML, Jodlowski TZ, Cutrell JB. Pharmacologic treatments for Coronavirus Disease 2019 (COVID-19): a review. JAMA. 2020; 323(18): 1824-36.

8. Zhai P, Ding Y, Wu X, Long J, Zhong Y, Li Y. The epidemiology, diagnosis and treatment of COVID-19. Int J Antimicrob Agents. 2020; 55(5): 105955.

9. Rogers JP, Chesney E, Oliver D, Pollak TA, McGuire P, Fusar-Poli P, et al. Psychiatric and neuropsychiatric presentations associated with severe coronavirus infections: a systematic review and meta-analysis with comparison to the COVID-19 pandemic. Lancet Psychiatry. 2020; 7(7): 611-27.

10. Guo Q, Zheng Y, Shi J, Wang J, Li G, Li C, et al. Immediate psychological distress in quarantined patients with COVID-19 and its association with peripheral inflammation: a mixed-method study. Brain Behav Immun. 2020; 88: 17-27.

11. Lovell N, Maddocks M, Etkind SN, Taylor K, Carey I, Vora V, et al. Characteristics, symptom management and outcomes of 101 patients with COVID-19 referred for hospital palliative care. J Pain Symptom Manage. 2020; S0885-3924(20): 30211-6.

12. Liguori C, Pierantozzi M, Spanetta M, Sarmati L, Cesta N, lannetta M, et al. Subjective neurological symptoms frequently occur in patients with SARS-CoV2 infection. Brain Behav Immun. 2020; 88: 11-6.

13. Singh JA, Beg S, Lopez-Olivo MA. Tocilizumab for rheumatoid arthritis: a Cochrane systematic review. J Rheumatol. 2011; 38(1): 10-20.

14. Harrold LR, John A, Reed GW, Haselkorn T, Karki C, Li Y, et al. Impact of Tocilizumab monotherapy on clinical and patient-reported qualityof-life outcomes in patients with rheumatoid arthritis. Rheumatol Ther. 2017; 4(2): 405-17.

15. Abers MS, Shandera WX, Kass JS. Neurological and psychiatric adverse effects of antiretroviral drugs. CNS Drugs. 2014; 28(2): 131-45.

16. Ginsberg DL. Azithromycin-induced psychotic depression and 
catatonia. Prim Psychiatry. 2006; 13(5): 22-6.

17. Dubovsky AN, Arvikar S, Stern TA, Axelrod L. The neuropsychiatric complications of glucocorticoid use: steroid psychosis revisited. Psychosomatics. 2012; 53(2): 103-15.

18. National Institute for Health and Care Excellence (NICE) in collaboration with NHS England and NHS Improvement. Managing COVID-19 symptoms (including at the end of life) in the community: summary of NICE guidelines. BMJ. 2020; 369: m1461.

19. Esteve Arríen A, Aguera Ortiz L, Manzano Palomo S. Manejo farmacológico de trastornos psicóticos en personas mayores con tratamiento de la infección por COVID19: interacciones y recomendaciones terapéuticas [Internet]. España; 2020. Disponible en:https://www. fesemi.org/sites/default/files/documentos/19.pdf

20. Khawam E, Khouli M, Pozuelo L. Treating acute anxiety in patients with Covid-19. Cleve Clin J Med. 2020

21. University of Liverpool. Interactions with experimental COVID-19 Therapies [Internet]. 2020. Disponible en: https://www.covid19druginteractions.org/

22. MedScape. Drug Interaction Checker [Internet]. 2020. Disponible en: https://reference.medscape.com/drug-interactionchecker

23. Huremović D. Psychiatry of Pandemics: a mental health response to infection outbreak. Springer Nature: Switzerland; 2019.

24. Zhang R, Wang X, Ni L, Di X, Ma B, Niu S, et al. COVID-19: melatonin as a potential adjuvant treatment. Life Sci. 2020; 250: 117583.

25. Zhang $\mathrm{K}$, Zhou X, Liu H, Hashimoto K. Treatment concerns for psychiatric symptoms in patients with COVID-19 with or without psychiatric disorders. Br J Psychiatry. 2020; 217(1): 351.

26. Lewis SR, Pritchard MW, Schofield-Robinson OJ, Alderson P, Smith AF. Melatonin for the promotion of sleep in adults in the intensive care unit. Cochrane Database Syst Rev. 2018; 5(5): CD012455.

27. Anttila SAK, Leinonen EVJ. A review of the pharmacological and clinical profile of mirtazapine. CNS Drug Rev. 2001; 7(3): 249-64.

28. Luykx JJ, Van Veen SMP, Risselada A, Naarding P, Tijdink JK, Vinkers $\mathrm{CH}$. Safe and informed prescribing of psychotropic medication during the COVID-19 pandemic. Br J Psychiatry. 2020; 217(3): 471-4.

29. Livingston RL, Zucker DK, Isenberg K, Wetzel RD. Tricyclic antidepressants and delirium. J Clin Psychiatry. 1983; 44(5): 173-6.

30. Kotfis K, Williams-Roberson S, Wilson JE, Dabrowski W, Pun BT, Ely EW. COVID-19: ICU delirium management during SARS-CoV-2 pandemic. Crit Care. 2020; 24(1): 176.

31. Baller EB, Hogan CS, Fusunyan MA, Ivkovic A, Luccarelli JW, Madva $E$, et al. Neurocovid: pharmacological recommendations for delirium associated with COVID-19. Psychosomatics. 2020.

32. Manjunatha N, Naveen Kumar C, Bada Math S. Mental health in the times of COVID-19 pandemic: Guidance for general medical and specialised mental health care settings. National Department of Psychiatry National Institute of Mental Health \& Neurosciences (NIMHANS). 2020.

33. Jernigan MG, Kipp GM, Rather A, Jenkins MT, Chung AM. Clinical implications and management of drug-drug interactions between antiretroviral agents and psychotropic medications. Ment Health Clin. 2013; 2(9): 274-85.

34. Bilbul M, Paparone P, Kim AM, Mutalik S, Ernst CL. Psychopharmacology of COVID-19. Psychosomatics. 2020.
Correspondencia:

Glauco Valdivieso Jiménez

Dirección: Av. 200 Millas S/N - Villa El Salvador. Lima, Perú. Teléfono: +51 944000518

Correo electrónico: glauco.valdivieso@unmsm.edu.pe

Recibido: 01 de julio de 2020

Evaluado: 20 de agosto de 2020

Aprobado: 31 de agosto de 2020

(c) La revista. Publicado por Universidad de San Martín de Porres, Perú. (c) ${ }_{\text {BY }}$ Licencia de Creative Commons Artículo en acceso abierto bajo términos de Licencia Creative Commons Atribución 4.0 Internacional. (http://creativecommons.org/licenses/by/4.0/)

ORCID iDs

Glauco Valdivieso-Jiménez 근 https://orcid.org/0000-0001-8561-6768 